\title{
ALCASYSTEM - Um Portal com Técnicas de Aprendizagem Ativa para Disciplinas da Área da Computação
}

\author{
Ronney Moreira de Castro, Sean Wolfgand Matsui Siqueira \\ Programa de Pós-Graduação em Informática (PPGI) \\ Universidade Federal do Estado do Rio de Janeiro (UNIRIO) \\ Av. Pasteur, 456 - Urca - Rio de Janeiro - RJ - Brasil \\ ronney.castro@uniriotec.br, sean@uniriotec.br
}

\begin{abstract}
Resumo. O presente artigo apresenta o ALCASYSTEM, um Portal com técnicas de Aprendizagem Ativa para Ensino de disciplinas da área da Computação. O objetivo é auxiliar docentes que queiram adotar essas técnicas revendo a forma como o conteúdo das diversas disciplinas da área da Computação é trabalhado, geralmente com uso de tecnologias de informação e comunicação, além de fornecer uma abordagem mais dialética e dialógica que permita maior envolvimento e desenvolvimento de uma ampla gama de habilidades por parte dos alunos. Espera-se que o Portal dissemine novas abordagens, estratégias e tecnologias, e possa contribuir para a promoção de uma melhoria no ensino da área da Computação.
\end{abstract}

\section{Cenário de uso}

A sociedade moderna é considerada digital, devido principalmente ao avanço e proliferação de tecnologias de comunicação e informação. Computadores, internet, celulares, entre outros estão disponíveis para uso pessoal e ocupam papel de destaque no cotidiano das pessoas. O perfil e o processo de aprendizado dos alunos mudaram [Loureiro; Messias 2016]. Bates (2015) destaca em seu livro "Teaching in a Digital Age", especialmente escrito sobre o contexto atual dos discentes, que o conhecimento envolve dois componentes interligados: i) conteúdo (fatos, evidências, ideias, princípios, descrições de processos), que a maioria dos docentes já possui e os quais têm uma boa compreensão; e ii) habilidades que correspondem a requisitos que as empresas de hoje necessitam. Estas habilidades incluem comunicação, capacidade de aprender de forma independente, ética e responsabilidade, trabalho em equipe e flexibilidade, pensamento crítico (criatividade, estratégias, originalidade, resolução de problemas), habilidades digitais, e gestão do conhecimento.

Nesse contexto, as instituições de ensino têm a responsabilidade de proporcionar a seus alunos oportunidades de aplicar e desenvolver o conhecimento, o que na área de Computação pode ser feito através dos laboratórios, oficinas, áreas de teste, salas de reuniões, salas de aula multimídia etc. [Barret et al. 2015] [Wilczynski 2015]. Entretanto, mesmo com todos esses recursos, as práticas educacionais nas aulas da área de Computação não têm conseguido provocar a criatividade e criticidade dos alunos que ficam desmotivados devido a vários fatores, tais como inadequações do professor, falta de entendimento dos conteúdos trabalhados, percepção de que não estão evoluindo na disciplina, entre outros [Genco; Hölttä-Otto; Seepersad, 2012] [Giraffa; Móra 2013].

Desde os anos sessenta, educadores têm questionado a forma como a educação vem sendo conduzida e buscado abordagens mais experimentais [Blikstein 2013]. O uso da Aprendizagem Ativa (AA) pode auxiliar de forma significativa neste sentido. A AA pode ser definida como "atividades instrucionais envolvendo os alunos a fazerem coisas 
VIII Congresso Brasileiro de Informática na Educação (CBIE 2019)

Anais dos Workshops do VIII Congresso Brasileiro de Informática na Educação (WCBIE 2019)

e pensar sobre o que estão fazendo" [Bonwell; Eison 1991] [Massey; Brown; Johnston 2005]. As técnicas de AA permitem, não somente que os alunos participem de atividades dentro e fora da sala de aula, mas também saiam de seu papel de apenas ouvintes com o professor sendo o centro das atenções e apenas aquele que é o detentor do conhecimento [Massey; Brown; Johnston 2005]. Um grande número de estratégias vem ganhando destaque, na promessa de obter a atenção dos alunos e auxiliá-los na aprendizagem, tais como salas de aula invertidas, uso de dinâmicas de grupo, jogos, entre outras [Goode et al. 2007] [Gudigantala 2013], em muitos casos com uso intensivo de tecnologias de comunicação e informação.

O uso de AA pode auxiliar os docentes que desejam promover novas experiências em suas aulas. Mas como encontrar a técnica de AA mais direcionada para determinado conteúdo e objetivo de aprendizagem? Faltam ferramentas que apoiem o compartilhamento de experiências sobre o uso de práticas de AA pelos docentes da área da computação. Muitas propostas dessas práticas são encontradas dispersas na literatura, ou seja, estão em anais de eventos, revistas, artigos encontrados em instituições de ensino, livros, sites etc., o que acaba também dificultando o acesso e uso das mesmas por docentes que queiram introduzi-las em suas aulas. O Portal ALCASYSTEM vem de encontro a esse problema possibilitando ao docente a busca por técnicas de AA para suas aulas em disciplinas da área da Computação.

\section{Desenvolvimento}

Com o objetivo de auxiliar o docente que deseja fazer a transição para um modelo didático na perspectiva da AA, foi desenvolvido um Portal Web que permite a inclusão, busca, seleção, classificação e recomendação de técnicas de AA. O Portal foi construído com base em três perspectivas: a disciplina na área de Computação, a técnica de AA utilizada e o fórum de publicação. As disciplinas de Computação, foram compiladas a partir dos referenciais de formação em Computação [Zorzo et al. 2017], onde as sugestões de conteúdos variados para os cursos da área (Ciência da Computação, Engenharia de Computação, Engenharia de Software, Licenciatura em Computação, Sistemas de Informação e Cursos Superiores de Tecnologia em Computação) foram integradas.

As técnicas de AA foram organizadas de acordo com a proposta de [Mitchell; Petter; Harris 2017], embora técnicas adicionais tenham sido encontradas que não correspondiam à lista original, resultando em novas entradas de técnicas de AA no sistema.

A base de dados foi elaborada através de um Mapeamento Sistemático da Literatura dos anais dos principais eventos do país relacionados à Informática na Educação: Workshops do CBIE (Congresso Brasileiro de Informática na Educação); SBIE (Simpósio Brasileiro de Informática na Educação); WEI (Workshop sobre Educação em Computação) e WIE (Workshop de Informática na Escola). Além dos eventos também foi incluída a RBIE (Revista Brasileira de Informática na Educação). Para que fossem encontradas as técnicas mais atuais utilizadas por docentes em todo Brasil, foram escolhidos para o mapeamento os artigos no período de 2013 a 2018.

Para a implementação do portal foram utilizados: 1) Linguagem JAVA versão 8 (https://www.oracle.com/java) - Linguagem de desenvolvimento; 2) IDE Eclipse versão Photon (https://www.eclipse.org/photon) - Utilizado para o desenvolvimento do Portal; 3) Servidor Apache TomCat versão 8 (http://tomcat.apache.org) - Servidor utilizado para 
VIII Congresso Brasileiro de Informática na Educação (CBIE 2019)

Anais dos Workshops do VIII Congresso Brasileiro de Informática na Educação (WCBIE 2019)

executar a aplicação Web; 4) Hibernate versão 5 (http://hibernate.org) - Framework para acesso ao banco de dados; 5) MySQL versão 5 (https://www.mysql.com) - Sistema Gerenciador de Banco de Dados adotado para armazenamento dos dados; 6) Apache Mahout versão 0.14 (https://mahout.apache.org) - Framework de aprendizado de máquina utilizado para as recomendações de usuários. O Portal está online, hospedado nos servidores da Amazon (https://aws .amazon.com), disponível de forma pública e pode ser acessado no endereço https://goo.gl/7HNUxj.

O Portal foi avaliado através de entrevistas semiestruturadas, no período de 16/04/2019 a 10/05/2019, por nove professores, sendo sete deles da área da Computação, um de ensino técnico e um da área de educação. O mesmo era apresentado a cada um dos entrevistados. Em seguida, eram passados dados de acesso (usuário e senha) e os participantes acessavam o Portal. Ao longo da utilização, eram realizadas perguntas sobre seu funcionamento, usabilidade, utilidade, entre outras. Algumas das falas desses docentes foram: "Muito bom", "É muito útil para auxiliar o cotidiano acadêmico", "A ferramenta está com boa usabilidade", "Certamente você deveria divulgar o seu sistema, depois que ele deixar de ser protótipo, com o pessoal da Comissão Especial de Educação da SBC", "Usaria uma ferramenta como essa todo dia", "Você já fez um trabalho de levantamento bibliográfico; que maravilha"; "Gostei, é uma coisa bacana", "Acho que sim ajudaria em buscar alguma coisa interessante para aplicar para os alunos".

Algumas falas também foram utilizadas para melhorias no Portal, tais como: "A tela inicial está muito careta.", "Talvez buscando por palavra seria melhor porque eu não teria que ler todos os artigos para ver a técnica", "Criar uma troca de experiências talvez seria mais interessante, porque ele falaria com os pares dele sobre as práticas pedagógicas", "Criação de um fórum para que os professores possam discutir sobre determinado trabalho". A tela inicial foi alterada segundo a sugestão de um dos docentes. Além disso, foi implementada a funcionalidade de busca por palavra-chave e está em desenvolvimento a criação de um fórum dentro do Portal. Já está disponível a opção para o docente incluir comentários sobre os trabalhos e os mesmos serem vistos pelos demais docentes usuários do Portal.

\section{Apresentação do Software}

Ao acessar o endereço do Portal ALCASYSTEM é exibida a tela inicial da figura 1.

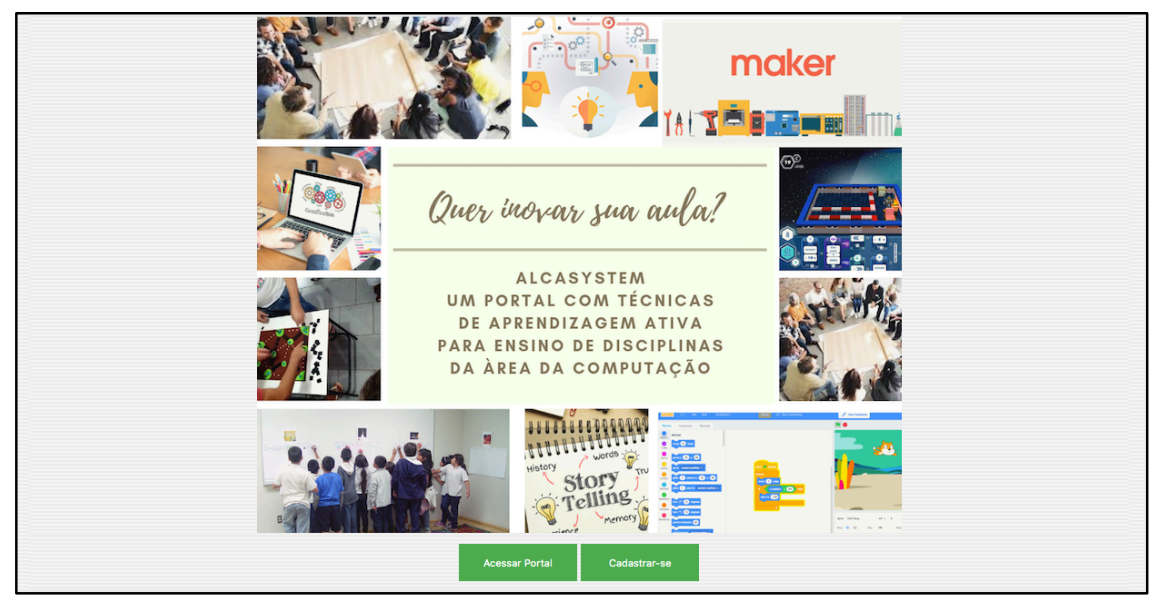

Figura 1. Tela de acesso ao ALCASYSTEM. 
VIII Congresso Brasileiro de Informática na Educação (CBIE 2019)

Anais dos Workshops do VIII Congresso Brasileiro de Informática na Educação (WCBIE 2019)

É necessário efetuar um cadastro para utilização do Portal. Para isso basta clicar no botão "Cadastrar-se" e preencher os dados da tela seguinte. Logo após, um e-mail é enviado ao usuário liberando seu acesso. Existem dois perfis de usuário: Administrador (possui acesso a todas as funcionalidades - Perfil exclusivo para os autores do artigo e/ou docentes que farão a manutenção do Portal) e Usuário (acesso somente a opção de recomendação - Perfil para utilização pelo docente).

O Menu de acesso do Administrador contém as opções: Categoria AA, Disciplinas, Evento, Técnicas AA e Trabalhos, todas com opções para incluir, alterar, excluir e consultar os dados. Menu Recomendação (cerne do Sistema), que permite ao professor fazer buscas pelas técnicas, visualizar e fazer download dos trabalhos relacionados às mesmas, além de poder armazenar os que mais interessam. O portal ainda permite efetuar a classificação dos trabalhos e tais informações serão utilizadas em um processo de recomendação para outros docentes com perfis similares. Além disso, os menus Informação (contém dados sobre o desenvolvimento do sistema) e Configurações (cadastro, listagem e liberação de usuários). A figura 2 exibe a tela inicial do Portal para o perfil Administrador.

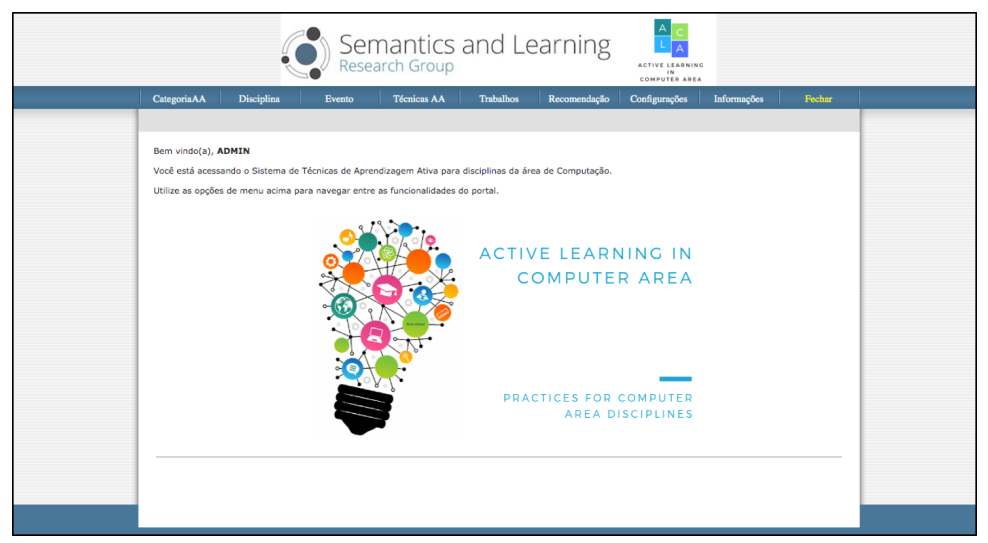

Figura 2. Tela inicial do Perfil Administrador.

A figura 3 exibe parte da tela de recomendação, onde o docente pode optar pelas opções Evento, Nível de Ensino, Disciplinas Envolvidas (um compilado das disciplinas dos Referenciais de Formação da SBC [Zorzo et al. 2017]), Categoria AA (com base no trabalho de [Mitchell; Petter; Harris 2017]), Técnicas, Palavra-Chave, ou então por qualquer combinação entre elas.

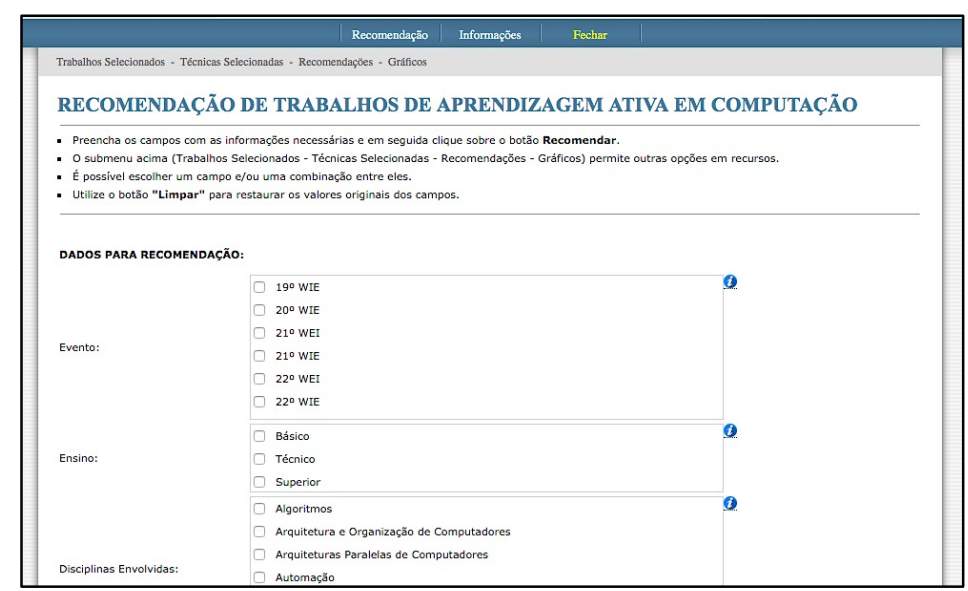

Figura 3. Opções para o Docente - Menu de Recomendação. 
VIII Congresso Brasileiro de Informática na Educação (CBIE 2019)

Anais dos Workshops do VIII Congresso Brasileiro de Informática na Educação (WCBIE 2019)

O docente pode também selecionar os trabalhos preferidos dentre os recomendados conforme figura 4. Aqueles que foram selecionados ficam armazenados em seu perfil, conforme figura 5. Basta acessar o sub-menu Trabalhos Selecionados

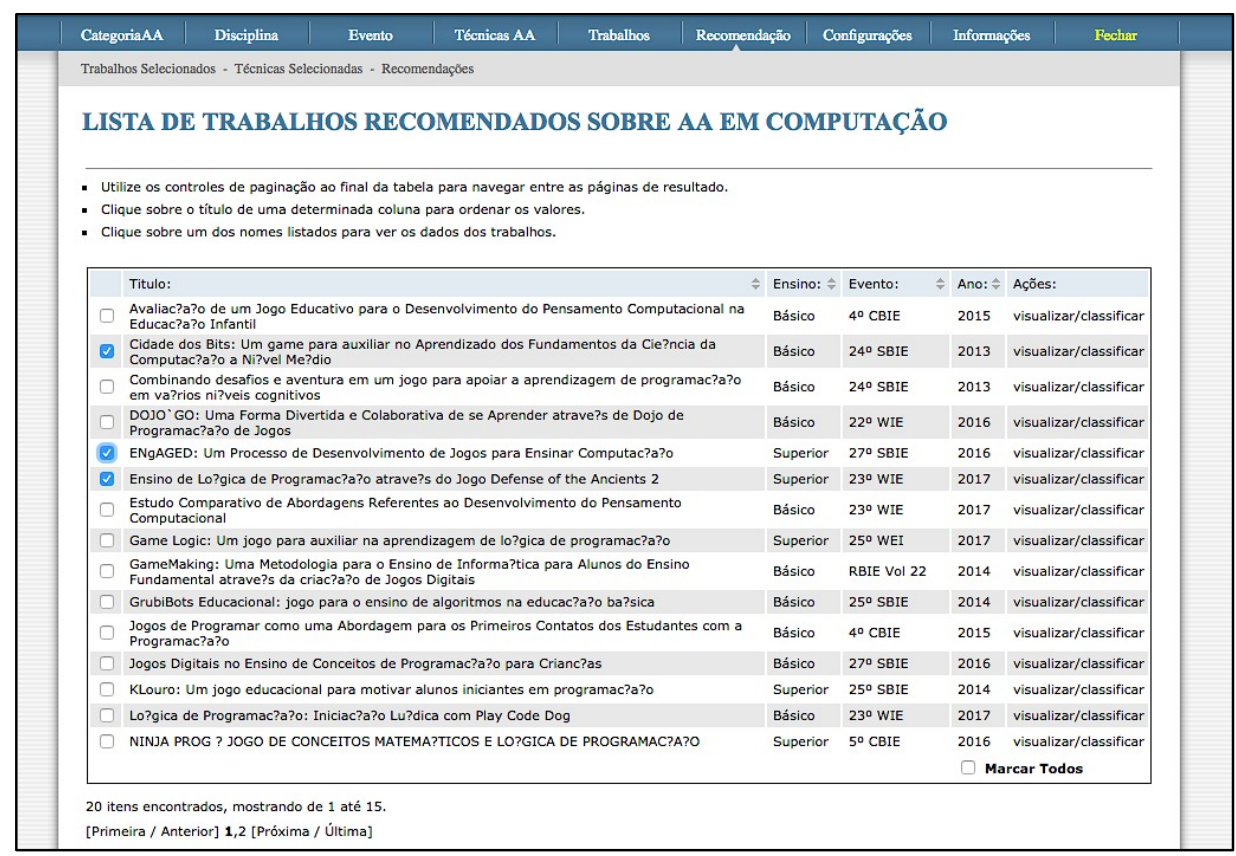

Figura 4. Lista de trabalhos recomendados.

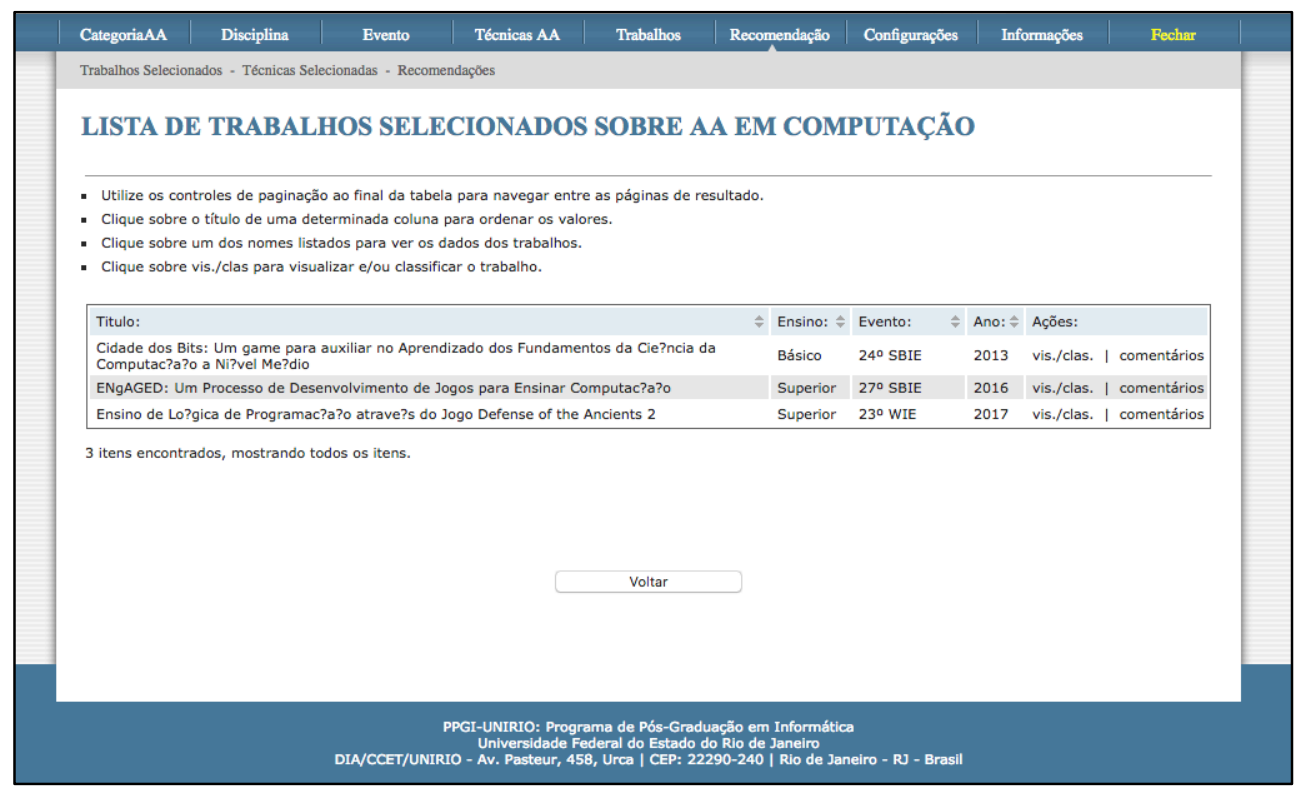

Figura 5. Lista de trabalhos escolhidos pelo usuário.

É possível também, a partir das telas exibidas nas figuras 4 e 5, classificar as técnicas e também fazer comentários, conforme figura 6 . 
VIII Congresso Brasileiro de Informática na Educação (CBIE 2019)

Anais dos Workshops do VIII Congresso Brasileiro de Informática na Educação (WCBIE 2019)

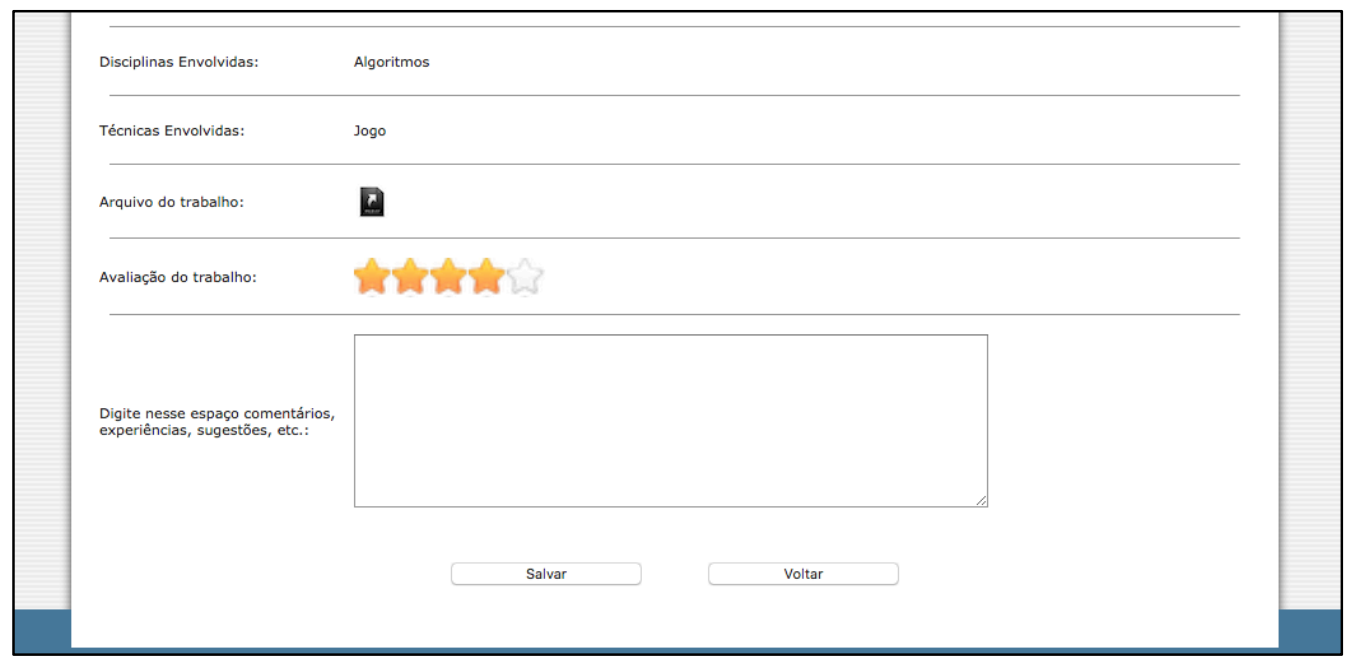

Figura 6. Tela de classificação/comentários do trabalho.

Os comentários podem ser visualizados por outros docentes, conforme figura 7 .

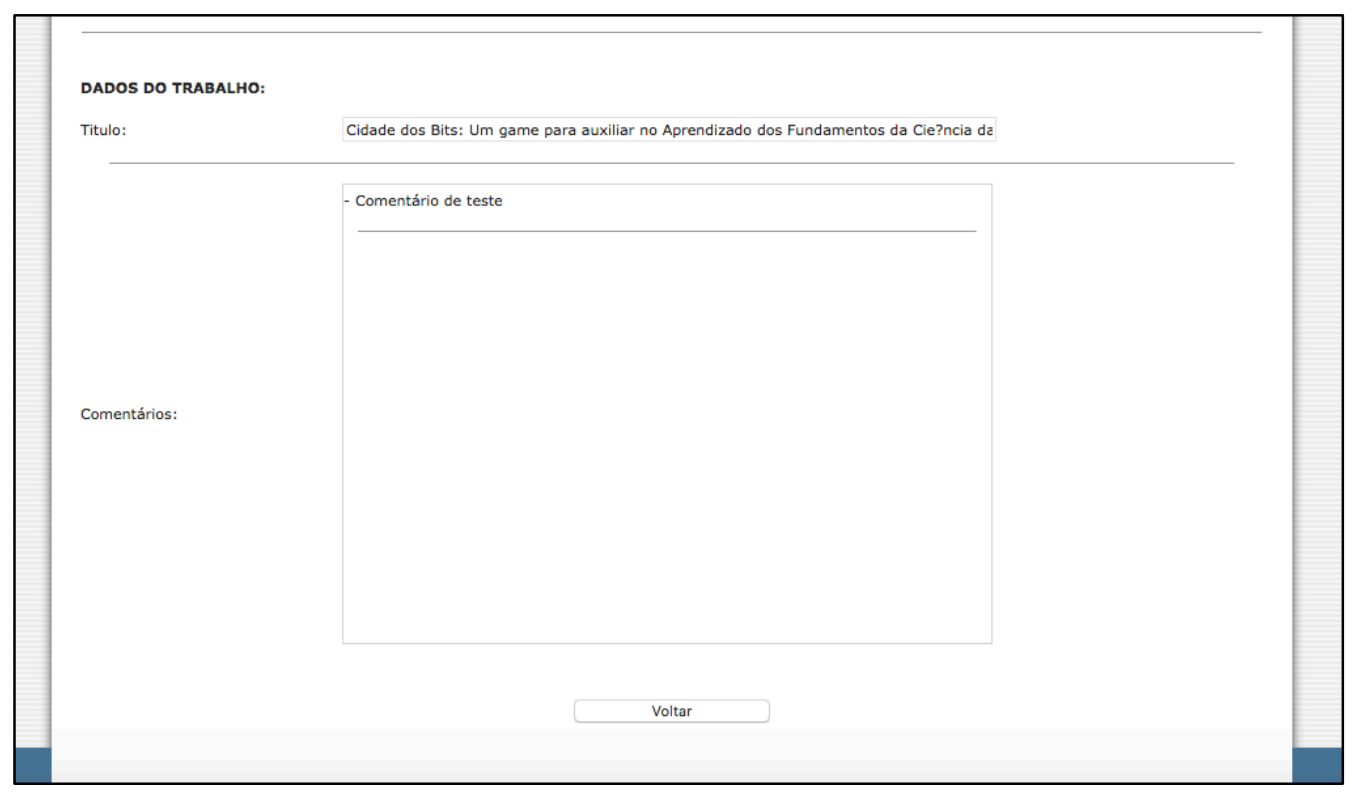

Figura 7. Lista de comentários de usuários para um determinado trabalho.

O link de acesso ao sistema é https://goo.gl/7HNUxj. Um vídeo demonstrativo do Portal pode ser visualizado pelo link https://youtu.be/iHohL6R2nlg. O software é de livre acesso podendo ser utilizado por docentes dos diferentes níveis de ensino.

\section{Considerações finais}

As aulas tradicionais tendem a não prender a atenção dos alunos. É necessário mudar este cenário e o uso da Aprendizagem Ativa passa a ser um diferencial e pode permitir um melhor aprendizado do conteúdo abordado nas aulas. As técnicas de AA, em geral, encontram-se dispersas na literatura o que acaba dificultando sua busca por parte dos docentes que querem implementá-las em suas aulas. Além disso, muitos docentes não possuem tempo disponível para realizar buscas ou se dedicar ao estudo de uma determinada técnica. Para auxiliar tais docentes foi desenvolvido o Portal ALCASYSTEM que possui atualmente, uma base contendo 285 trabalhos retirados dos 
VIII Congresso Brasileiro de Informática na Educação (CBIE 2019)

Anais dos Workshops do VIII Congresso Brasileiro de Informática na Educação (WCBIE 2019)

principais eventos de Informática e Educação e Ensino de Computação no Brasil. O Portal permite a recomendação, seleção e classificação de técnicas de AA.

O Portal foi validado por nove docentes através de entrevistas semiestruturadas. De forma geral, o mesmo foi bem avaliado, com destaque para falas de alguns docentes que relataram ser uma ótima forma de divulgação sobre técnicas alternativas de ensino, de fazer com que as técnicas contidas nos trabalhos dos eventos não sejam utilizadas apenas para fins de número de publicações, mas para utilização dos maiores interessados nelas: os docentes. Constatou-se também alguns problemas como o fato do professor ter que ler o artigo para utilizar uma técnica. Como trabalho futuro pode-se elaborar resumos das técnicas e colocá-las em formato de cases de estudo para direcionar melhor e mais rapidamente o uso das técnicas. Alguns docentes também sugeriram que o Portal fosse transformado em uma ferramenta colaborativa, com um fórum para debate entre os professores, o que também poderá ser realizado como trabalho futuro.

\section{Referências}

Barrett, T. W., Pizzico, M. C., Levy, B., Nagel, R. L. (2015). A Review of University Maker Spaces A Review of University Maker Spaces Introduction. 122nd ASEE Annual Conference and Exposition, 1-16.

Bates T. (2016). Teaching in a digital age. In: BC Open Textbooks. BCcampus. Available via BCcampus Services. Disponível em:<http://opentextbc.ca/teachinginadigitalage $>$. Acesso em 29 Jan. 2017.

Blikstein, P. (2013). Digital Fabrication and "Making" in Education: The Democratization of Invention. FabLabs: Of Machines, Makers and Inventors, 1-21.

Bonwell, C. C., Eison, J. A. (1991). Active learning: Creating excitement in the classroom. Association for the Study of Higher Education, Washington, DC.

Genco, N., Hölttä-Otto, K., Seepersad, C. C. (2012). An experimental investigation of the innovation capabilities of undergraduate engineering students. Journal of Engineering Education, 101(1), 60.

Giraffa, L. M. M., Móra, M. C. (2013). Evasão e Disciplina de Algoritmo e Programação: Um Estudo a partir dos Fatores Intervenientes na Perspectiva do Aluno. III Conferencia Latinoamericana Sobre El Abandono En La Educación Superior, 1-10.

Goode, S., Willis, R. A., Wolf, J. R., Harris, A. L. (2007). Enhancing IS education with flexible teaching and learning. Journal of Information Systems Education, 18 (3).

Gudigantala, N. (2013). An active learning approach to teaching undergraduate introduction to MIS Course. Paper presented at the 19th Americas Conference on Information Systems, Chicago, Illinois.

Loureiro, A., Messias, I. (2016). Competences and Learning Profiles of Digital Age's Students . In M. Pinheiro, D. Simões (Eds.), Handbook of Research on Engaging Digital Natives in Higher Education Settings - chapter08. Hershey, PA: IGI Global.

Massey, A. P., Brown, S. A., Johnston, J. D. (2005). It's All Fun and Games. Until Students Learn. Journal of Information Systems Education, 16 (1). 
VIII Congresso Brasileiro de Informática na Educação (CBIE 2019)

Anais dos Workshops do VIII Congresso Brasileiro de Informática na Educação (WCBIE 2019)

Mitchell, A., Petter, S., Harris, A. (2017). Learning By Doing: Twenty Successful Active Learning Exercises for Information Systems Courses. Journal of Information Technology Education: Innovations in Practice, 16(3), 21-46.

Zorzo, A. F., Nunes, D., Matos, E., Steinmacher, I., Leite, J., Araujo, R. M., Correia, R., Martins, S. (2017). Referenciais de Formação para os Cursos de Graduação em Computação. Sociedade Brasileira de Computação (SBC). 153p, 2017.

Wilczynski, V. (2015). Academic maker spaces and engineering design. American Society for Engineering Education. 